\title{
Development of the Exact Exchange Scheme Using a Basis Set Framework
}

\author{
J. J. FERNÁNDEZ, C. TABLERO, P. WAHNÓN \\ Departamento TEAT. ETSI Telecomunicación, Ed.A-034, Unizersidad Politécnica de Madrid, Ciudad \\ Universitaria s/n 28040 Madrid, Spain
}

\begin{abstract}
A combination of the exact exchange (EXX) scheme for the approximation of the exchange term within the density functional theory is presented. For this task we have developed it and implemented in the atom code program. As will be discussed in this article, the use of this approach has the advantages of using a simple, but efficient, calculation numerical code and the refinement of the EXX method for the exchange part within the Khon-Sham formalism. We present the development of the EXX theory within an atomic scheme for energy calculations. Results for several atomic systems are presented. We found a remarkable improvement in the energies of all the systems studied compared with the local density approximation and generalized gradient approximation approaches. $\quad 2002$ Wiley Periodicals, Inc. Int J Quantum Chem 91: $157-164,2003$
\end{abstract}

Key words: exact energy determination; exact exchange approach; local density approximation; OEP methods

\section{Introduction}

D ensity functional theory (DFT) has made a great impact on the calculation of groundstate properties of atoms, molecules, and solids, in

Correspondence to: J. J. Fernández; e-mail: Julio@etsit.upm.es Contract grant sponsor: Plan Nacional de I+D of Spain and FEDER.

Contract grant numbers: TIC2000-1339-C02-02; 2FD97-0332C03-01.

Contract grant sponsor: European Commission.

Contract grant number: ENK6 CT200 00310 . particular, for predicting quantitative material properties obtained from total energy calculations [1-2].

However, this theory has several deficiencies, one of them being the incorrect treatment of the exchange and correlation terms. In their early works, Kohn and Sham proposed to represent the exchange and correlation term by a functional expression based on the study of the homogeneus electron gas: this is the so-called local density approximation (LDA) approach [3-5]. Later on, several authors proposed new functional forms for the exchange term based on the use of the gradient in 
the density to improve the lack of the exchange functional nonlocality. All these approximations are included in the so-called generalized gradient approximation (GGA) functionals [6-8]. Both methods have deficiencies with the estimation of binding molecules energies or lattice parameters in solids and especially with the determination of the highest occupied molecular orbital (HOMO)-lowest unoccupied molecular orbital (LUMO) energy differences in molecules and the band-gap values of the semiconductors. In the atoms, the use of the DFT does not lead to a good description of the exchange potential in the valence region. This makes the atoms present an incorrect behavior, in the form of the exchange potential in the valence region not yielding a Rydberg series and having a potential that falls off too quickly at long distances instead of reaching the desired $1 / r$ asymptotic behavior.

Several authors [9-13] have developed a new formalism for approximating the exchange term with the goal of introducing a term that is dependent on orbitals and not on the density, as are the LDA and GGA approaches. There are some applications of this concept within the Khon-Sham theory, but many of these include very heavy restrictions. Some of them are applicable only to systems having spherical symmetry [9]. Others, such as the Krieger-Li-Iafrate (KLI) approach, consider all the energy differences between the Kohn-Sham eigenvalues as constant and get a potential that is based on the overall average of this quantity [10,11]. The latter approaches are nowadays presented as the OEP methods [9], because they follow the original method of the optimized effective potential for atoms. In the framework of this context, in very recent years some authors have developed and implemented (mainly in planewave codes) a new exchange functional based on building up an exchange density [14-16], the exact exchange method (EXX) and getting the exchange potential by integration of this charge distribution.

The main purpose of the work in this article is to present results obtained in the calculation of the electronic structure of several atomic systems, by means of a localized basis set representation scheme of the EXX [14-16] approach, to calculate the exchange term within the Khon-Sham formalism [1].

For this task we present a detailed analysis of the basis set used to represent the exchange density in atoms, as a first step for the subsequent use of this scheme the EXX method within the SIESTA formalism [20]. In our current development, we have combined the EXX formalism with an atomic numerical code "atom," which is used in SIESTA [20] and used it to study the convergence on the atomic properties when improving the basis set.

We present the mathematical formalism needed for implementing the calculation of the exchange term using the EXX approach in the atomic code used.

We also present the results obtained using this method for several atoms. The calculations have been carried out mainly with two purposes. The first is to look for a good basis set for the expansion of the exchange density and the other, the need to get a good description of the atoms.

\section{EXX Formalism and Computational Details}

In this work we present an application of the formalism originally developed by A. Görling et al. [14-16] for the calculation of the exchange term. As in the SIESTA [17] code, we use numerical local orbitals for the building of the exchange operator. For the expansion of the exchange density that should be understood as a distribution of density yielding the exchange potential, denoted as $\rho_{x}(\mathbf{r})$, we use a basis of numerical functions. These functions will contain a radial part taking the form of a Slater function.

$$
f_{i}(\mathbf{r})=r^{n_{i}} \cdot e^{-\xi_{i} r} Z_{l_{i}, m_{i}}(\theta, \phi)
$$

With $i$ being the number of the basis function, and $Z_{l_{i} m_{i}}$ being a real spherical harmonic [18].

To generate the exchange potential the mathematical development starts by assuming that the exchange potential obeys the OEP integral equation [9-12] in which we represent the integral kernel of the term by using the static response operator for the system being studied [3]:

$$
\int d \mathbf{r}^{\prime} X\left(\mathbf{r}, \mathbf{r}^{\prime}\right) v_{x}\left(\mathbf{r}^{\prime}\right)=\sum_{a} \sum_{s} \phi_{a}(\mathbf{r}) \phi_{s}(\mathbf{r})\left\langle\phi_{s}\left|v_{x}^{N L}\right| \phi_{a}\right\rangle
$$

where $a$ denotes an occupied state, $s$ an unoccupied one, and $v_{x}^{N L}$ is the so-called nonlocal exchange potential, because it takes the form of a potential 
TABLE I

Orbital energies for different atoms. ${ }^{a}$

\begin{tabular}{|c|c|c|c|c|c|c|}
\hline & LDA & GGA & EXX case I & EXX case II & EXX case III & $\begin{array}{c}\text { Experimental } \\
\text { values [21] }\end{array}$ \\
\hline \multicolumn{7}{|c|}{ Carbon } \\
\hline 1s & -19.8957 & -20.0840 & -20.46058 & -20.41471 & -20.5208 & -20.79 \\
\hline $2 s$ & -1.0019 & -1.0098 & -1.14861 & -1.24260 & -1.24262 & -20.71 \\
\hline $2 p$ & -0.3985 & -0.3887 & -0.43360 & -0.62664 & -0.72602 & $-1.48 /-1.45$ \\
\hline $3 d$ & 0.0000 & 0.0000 & -0.03433 & -0.03834 & -0.04567 & $-0.81 / 0.00$ \\
\hline $4 f$ & 0.0000 & 0.0000 & -0.012312 & -0.01423 & -0.01612 & \\
\hline \multicolumn{7}{|c|}{ Gallium } \\
\hline $1 \mathrm{~s}$ & -740.3424 & -741.8186 & -741.7202 & -742.2801 & -742.2800 & -762.79 \\
\hline $2 s$ & -90.4018 & -90.5586 & -90.5606 & -90.9074 & -90.9077 & -95.144 \\
\hline $2 p$ & -80.1869 & -80.2321 & -80.2342 & -80.6572 & -80.6476 & -84.058 \\
\hline $3 s$ & -10.4823 & -10.5324 & -10.5344 & -11.2021 & -11.2029 & -11.720 \\
\hline $3 p$ & -7.1684 & -7.1831 & -7.1851 & -7.4020 & -7.4041 & -7.610 \\
\hline $3 d$ & -1.4715 & -1.4533 & -1.4557 & -1.3140 & -1.3144 & -1.354 \\
\hline $4 s$ & -0.6563 & -0.6401 & -0.6443 & -0.7832 & -0.7833 & \\
\hline $4 p$ & -0.2037 & -0.1922 & -0.1950 & -0.3028 & -0.3020 & \\
\hline $4 d$ & 0.0000 & 0.0000 & 0.0000 & 0.0000 & -0.0698 & \\
\hline $4 f$ & 0.0000 & 0.0000 & 0.0000 & 0.0000 & -0.0075 & \\
\hline \multicolumn{7}{|c|}{ Arsenic } \\
\hline $1 \mathrm{~s}$ & -846.6746 & -848.1486 & -848.1495 & -868.1495 & -868.1497 & -872.57 \\
\hline $2 s$ & -106.1863 & -106.3525 & -106.3534 & -110.3534 & -110.3536 & -112.27 \\
\hline $2 p$ & -95.0560 & -95.1048 & -95.1057 & -98.1057 & -98.1066 & -99.93 \\
\hline $3 s$ & -13.4608 & -13.5185 & -13.5195 & -14.5195 & -14.5208 & -15.05 \\
\hline $3 p$ & -9.7027 & -9.7217 & -9.7227 & -10.5227 & -10.5224 & -10.75 \\
\hline $3 d$ & -3.0848 & -3.0667 & -3.0680 & -3.9080 & -3.9086 & -4.56 \\
\hline $4 s$ & -1.0474 & -1.0329 & -1.0367 & -1.0767 & -1.0778 & \\
\hline $4 p$ & -0.3954 & -0.3844 & -0.3880 & -0.3911 & -0.3931 & \\
\hline $4 d$ & 0.0000 & 0.0000 & 0.0000 & 0.0000 & 0.0662 & \\
\hline $4 f$ & 0.0000 & 0.0000 & 0.0000 & 0.0000 & 0.0063 & \\
\hline \multicolumn{7}{|c|}{ Titanium } \\
\hline 1s & -355.5541 & -355.4822 & -357.5237 & -358.1237 & -358.1237 & -362.9091 \\
\hline $2 s$ & -39.1280 & -39.0241 & -40.4443 & -40.9443 & -40.9449 & -41.9091 \\
\hline $2 p$ & -32.6101 & -32.5964 & 34.4950 & -35.250 & -35.251 & -33.351 \\
\hline $3 s$ & -4.6110 & -4.5425 & -4.8590 & -5.1597 & -5.1598 & -5.5012 \\
\hline $3 p$ & -2.9001 & -2.8506 & -3.2350 & -3.2350 & -3.2348 & -3.2110 \\
\hline $4 s$ & -0.3101 & -0.3251 & -0.5490 & -0.5490 & -0.5491 & -0.5460 \\
\hline $4 p$ & -0.1041 & -0.1212 & -0.2717 & -0.2717 & -0.2790 & -0.2999 \\
\hline $3 d$ & -0.3180 & -0.4132 & -0.7709 & -0.7718 & -0.7718 & \\
\hline $4 d$ & & & -0.2740 & -0.0747 & -0.2714 & \\
\hline $4 f$ & & & -0.0123 & -0.0125 & -0.0122 & \\
\hline
\end{tabular}

${ }^{a}$ We represent values obtained in LDA, GGA, and three different cases for the EXX potential. Basis parameters for the exchange density expansion in the three cases can be found in Table II.

arising from a nonlocal distribution of charge. The $\phi_{\alpha}^{\prime}$ functions denote the Khon-Sham orbitals. Finally $X\left(\mathbf{r}, \mathbf{r}^{\prime}\right)$ denotes the static response function of an electronic system and has its usual form [1]:

$$
X\left(\mathbf{r}, \mathbf{r}^{\prime}\right)=\sum_{\mathrm{a}} \sum_{\mathrm{s}} \frac{\phi_{\mathrm{a}}\left(\mathbf{r}^{\prime}\right) \phi_{\mathrm{s}}\left(\mathbf{r}^{\prime}\right)}{\epsilon_{\mathrm{a}}-\epsilon_{\mathrm{s}}} \phi_{\mathrm{s}}(\mathbf{r}) \phi_{\mathrm{a}}(\mathbf{r})
$$

After solving these equations for the exchange potential we get the expansion coefficients for the exchange density. The exchange potential $\mathrm{v}_{x}(\mathrm{r})$ is then obtained through the integration of this quantity:

$$
\rho_{x}(\mathbf{r})=\sum_{i} C_{i} f_{i}(\mathbf{r})
$$


TABLE II

Parameters for the Slater basis functions used in the expansion of the exchange density for $\mathrm{C}, \mathrm{Ga}, \mathrm{As}$, and $\mathrm{Ti}$ atoms. ${ }^{\mathrm{a}}$

\begin{tabular}{|c|c|c|c|}
\hline & First case & Second case & Third case \\
\hline \multicolumn{4}{|l|}{ Carbon } \\
\hline Maximum / in expansion & 0 & 3 & 6 \\
\hline $\begin{array}{l}\text { No. of functions for each } / \\
\text { from } /=0 \text { to } / \max \end{array}$ & 2 & $3,2,2,3$ & $3,3,3,2,2,2$ \\
\hline $\begin{array}{l}\text { No. of different exponents in } \\
\text { Slaters }\end{array}$ & 5 & 4 & 4 \\
\hline Total of basis functions used & 10 & 20 & 40 \\
\hline Values for the exponents & $0.10,0.14,0.30,1.50,2.50$ & $0.14,0.16,0.32,1.27$ & $0.14,0.16,0.32,1.27$ \\
\hline \multicolumn{4}{|l|}{ Gallium } \\
\hline Maximum / in expansion & 0 & 3 & 7 \\
\hline $\begin{array}{l}\text { No. of functions for each } / \\
\text { from } /=0 \text { to } / \max \end{array}$ & 2 & $3,2,2,3$ & $3,3,3,2,2,2,1$ \\
\hline $\begin{array}{l}\text { No. of different exponents in } \\
\text { Slaters }\end{array}$ & 5 & 4 & 5 \\
\hline Total of basis functions used & 10 & 20 & 40 \\
\hline Values for the exponents & $0.10,0.14,0.30,1.50,2.50$ & $0.14,0.16,0.32,1.27$ & $0.14,0.16,0.32,1.27,1.50$ \\
\hline \multicolumn{4}{|l|}{ Arsenic } \\
\hline Maximum / in expansion & 0 & 3 & 7 \\
\hline $\begin{array}{l}\text { No. of functions for each } / \\
\text { from } /=0 \text { to } / \max \end{array}$ & 2 & $3,2,2,3$ & $3,3,3,2,2,2,1$ \\
\hline $\begin{array}{l}\text { No. of different exponents in } \\
\text { Slaters }\end{array}$ & 5 & 4 & 5 \\
\hline Total of basis functions used & 10 & 20 & 52 \\
\hline Values for the exponents & $0.10,0.14,0.30,1.50,2.50$ & $0.14,0.16,0.32,1.27$ & $0.13,0.21,0.35,1.37,1.51$ \\
\hline \multicolumn{4}{|l|}{ Titanium } \\
\hline Maximum / in expansion & 0 & 3 & 10 \\
\hline $\begin{array}{l}\text { No. of functions for each } / \\
\text { from } I=0 \text { to } / \max \end{array}$ & 2 & $3,2,2,3$ & $4,3,3,3,2,2,2,1,1$ \\
\hline $\begin{array}{l}\text { No. of different exponents in } \\
\text { Slaters }\end{array}$ & 5 & 4 & 5 \\
\hline Total of basis functions used & 10 & 20 & 40 \\
\hline Values for the exponents & $0.10,0.14,0.30,1.50,2.50$ & $0.14,0.16,0.32,1.27$ & $0.14,0.16,0.32,1.27,1.50$ \\
\hline
\end{tabular}

a Table shows the maximum / used in each case, the total number of functions and the value for the exponents.

$$
v_{x}(\mathbf{r})=\int \frac{\rho_{x}\left(\mathbf{r}^{\prime}\right)}{\left|\mathbf{r}-\mathbf{r}^{\prime}\right|} d \mathbf{r}^{\prime}
$$

Equation (4) has to be solved consistently with the symmetry of the problem with which we are dealing. So we integrate the electronic density in a very fine radial mesh to obtain the exchange potential with the correct $1 / r$ asymptotic behavior.

\section{Results}

In this section we present the results obtained when using the EXX approach for the exchange term in the calculation of several atoms $(\mathrm{H}, \mathrm{C}, \mathrm{Ti}$, $\mathrm{Ga}, \mathrm{As} . .$. ) and an analysis of the basis used for the development of the exchange density, as well as the generation of pseudopotentials for further use in the study of more complicated systems, such as solids and molecules.

As stated earlier in this article, the use of the EXX potential in atoms allows us to recover some properties that are lost when using the LDA or GGA approaches for the exchange term. For this purpose we carry out an analysis of the convergence of the basis set used for the exchange density expansion.

When we apply the formalism described in previous sections to the $\mathrm{C}, \mathrm{Ti}, \mathrm{Ga}, \mathrm{As}, \ldots$ atoms we find 

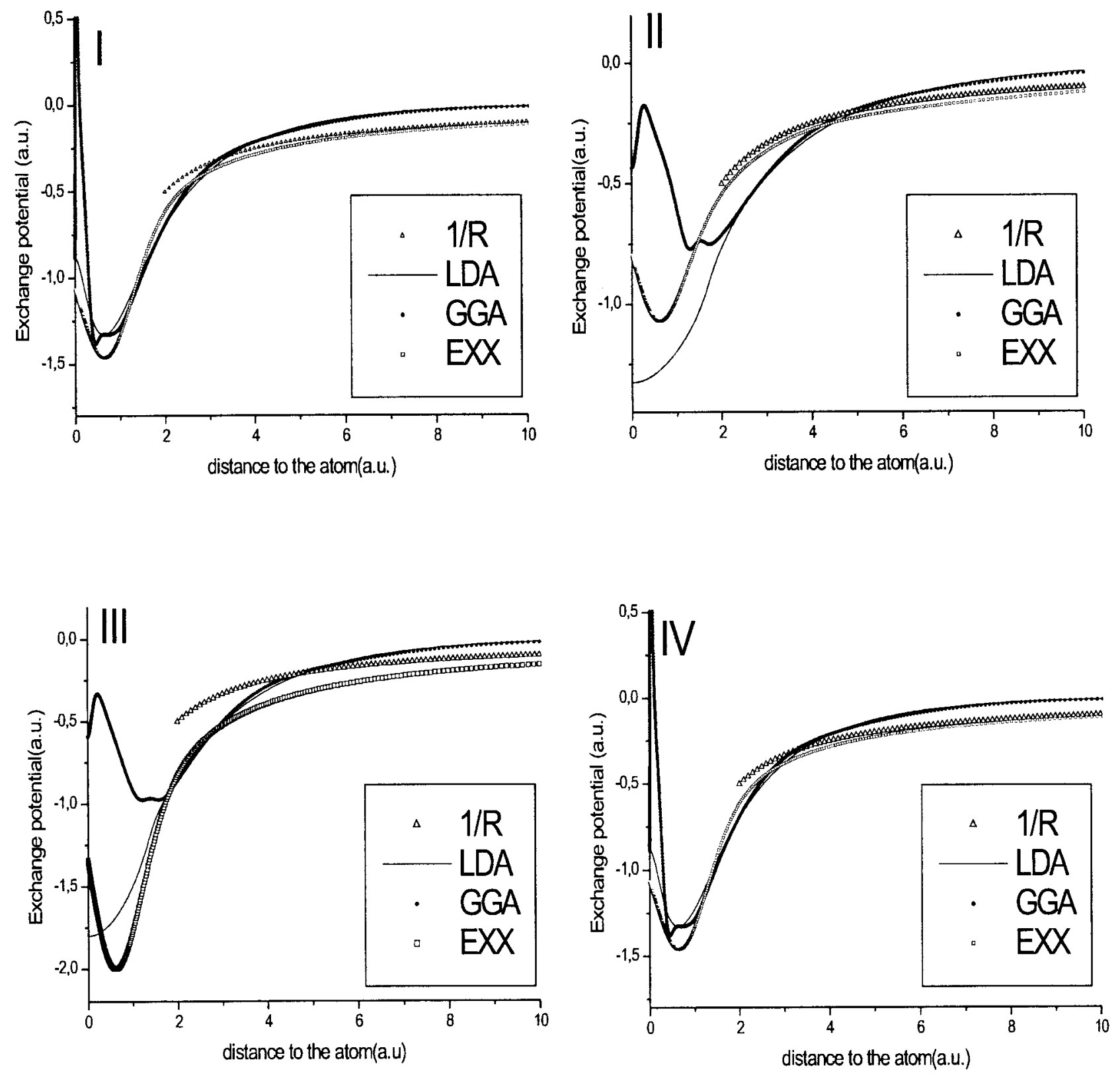

FIGURE 1. Radial part of the exchange potential obtained using different approaches for the exchange term: (I) carbon atom, (II) gallium atom, (III) arsenic atom, and (IV) titanium atom. In each figure we represent the LDA exchange potential, the GGA exchange potential, and the EXX exchange potential obtained using the parameters in the third case in Table II. Dotted line represents the right asymptotic behavior at long distances (1/r).

that the description of the quantities, such as orbital energies, exchange energy, and the total energy of the system, is much improved when compared with results obtained by the usual LDA and GGA procedures.

In Table I we analyze three different cases for the calculation of the orbital energies when using the EXX potential. These three different cases arise when the basis for the development of the exchange density is different. In this table, as a reference, we also include the orbital values obtained by using a typical LDA, and Perdew-Burke-Ermzerhoff (PBE-GGA) functionals (both of them obtained in a numerical calculation) and experimental values obtained from the literature. 


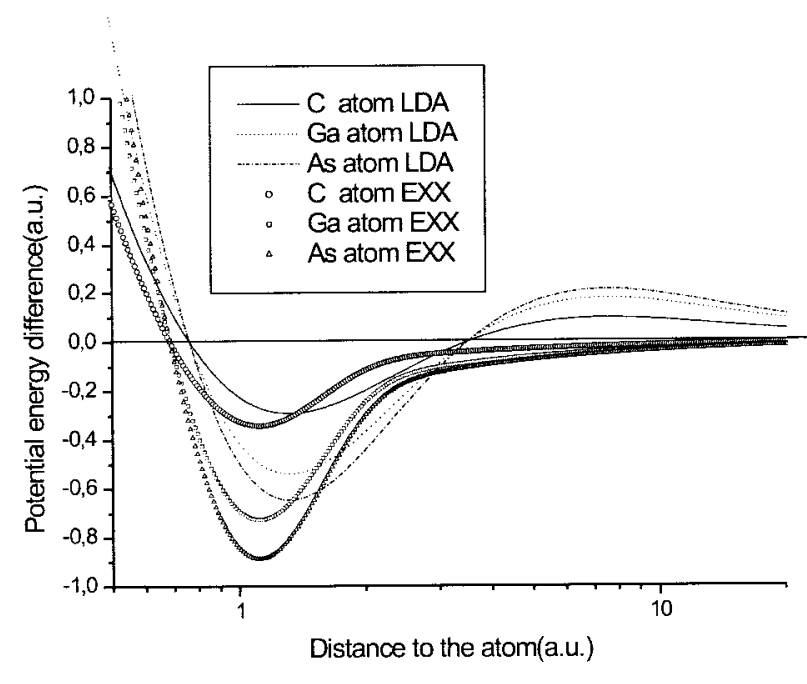

FIGURE 2. Representation of the difference between the radial part of the exchange potential and the $(1 / r)$ term for the $\mathrm{C}, \mathrm{Ga}$, and As atoms. All values are in atomic units.

In Table II we present the parameter values used in each of these cases. In these cases we increase the size of the basis until we get a good convergence in the total energy values. In the first row of this table we show the maximum value of $l$ used for the expansion of the potential. In the second row we show values for the number of functions (radial parts) used for each one of the $l$ terms used in the expansion of the exchange density in terms of the basis functions. The third row shows the number of parameters used in the Slater part of the basis function. The last row shows the values for the exponents used in the expansion.

Figure 1 compares three forms for the exchange potential for C, Ga, As, and $\mathrm{Ti}$ atoms. The first is obtained by using the LDA scheme, the second by using the GGA scheme, and the third represents the result when using the EXX formalism. The expansion parameters are displayed in Table I, in the last row.

The main characteristic of the LDA and GGA exchange potential is that at long distances it decreases very fast, going down to zero. As can be seen in Figure 1, the EXX exchange potential has the correct asymptotic behavior. Our potential also shows some differences in the valence region, leading to a better description of the inner core orbitals (Table I). This allows us to have a good description for outer levels, such as the $3 d$ and the $4 f$. In Figure 2 we see that the EXX potential reaches the asymptotic $1 / r$ behavior compared with the LDA ex- change potential, which does not reach this asymptotic behavior and falls off very quickly to zero.

Gallium and arsenic atoms present a more complicated electronic structure than does the $\mathrm{C}$ atom. In the calculations for these atoms we have considered levels up to $3 d$ to be included in the inner core region. We have also treated levels $4 s, 4 p, 4 d$, and $4 f$. as outer levels, to be included explicitly in the calculation.

Our results show a good improvement for the orbital energies, as can be seen in the comparison of the values presented in columns 5 and 6 of Table I. As in the case of the carbon atom, we recover the existence of a Rydberg series in both the gallium and arsenic atoms.

In the case of titanium we have incorporated the $3 d$ levels explicitly into the calculation as valence levels. This leads to the need to improve the basis for the expansion of the exchange density up to higher values of the angular momentum $l$. As shown in Table II, the maximum angular momentum $l$ required to obtain a good convergence (see Formula 2) is increased up to value of 10 .

In Figure 1 we can see how the description of the inner orbitals is greatly improved only with the use of a few $l$ values, but we have to increase this value considerably when we want to describe the semioccupied $3 d$ level correctly. In this case we also maintain the correct asymptotic behavior for the potential, even at long distances $(1 / r$ asymptotic behavior).

Table III shows values for the total energies for several atoms ranging from $Z=2(\mathrm{He})$ to $Z=10$ (Ne). Columns 1, 2, 3, and 4 show the value of the total energy obtained using different approaches for the exchange potential, such as the KLICs [19], exchange-correlation LDA (xcLDA) [19], Becke, Lee, Yang, and Paar (BLYP) [19], and PerdewWang (PW91) [19] approaches. Column 5 shows values obtained as a quantum chemistry calculation [20] and last column shows the experimental levels taken from Ref. 21. As can be seen in this table, the EXX results are in good agreement with the ones obtained by other authors using other techniques for calculating the exchange term [10-13] and with the experimental values.

Figure 3 represents the difference between the total energy calculated with the EXX method and the experimental values [21], versus the size of the basis set. We have represented five different sizes of basis set in this figure, the last three presented in Table II, for $\mathrm{Ti}, \mathrm{As}, \mathrm{Ga}$, and $\mathrm{C}$ atoms. This figure 
TABLE III

Total energies (ev) calculated using the LDA, GGA, and EXX exchange potentials. ${ }^{\text {a }}$

\begin{tabular}{lrrrrrrr}
\hline & KLICS [19] & XCLDA [19] & BLYP [19] & PW91 [19] & QC [20] & EXX & Exact [21] \\
\hline $\mathrm{He}$ & 2.9033 & 2.8346 & 2.9071 & 2.9000 & 2.9049 & 2.9035 & 2.9037 \\
$\mathrm{Li}$ & 7.4829 & 7.3433 & 7.4827 & 7.4342 & 7.4743 & 7.4900 & 7.4781 \\
$\mathrm{Be}$ & 14.6651 & 14.4465 & 14.6615 & 14.6479 & 14.6657 & 14.6662 & 14.6674 \\
$\mathrm{~B}$ & 24.6564 & 24.3525 & 24.6458 & 24.6299 & 24.6515 & 24.6566 & 24.6539 \\
$\mathrm{C}$ & 37.8490 & 37.4683 & 37.8430 & 37.8265 & 37.8421 & 37.8320 & 37.8450 \\
$\mathrm{~N}$ & 54.5905 & 54.1344 & 54.5932 & 54.5787 & 54.5854 & 54.5602 & 54.5893 \\
$\mathrm{O}$ & 75.0717 & 74.5248 & 75.0786 & 75.0543 & 75.0613 & 75.0730 & 75.067 \\
$\mathrm{~F}$ & 99.7302 & 99.1112 & 99.7316 & 99.7316 & 99.7268 & 99.7541 & 99.734 \\
$\mathrm{Ne}$ & 128.9202 & 128.2297 & 128.9466 & 128.9466 & 128.9927 & 128.9801 & 128.939 \\
\hline
\end{tabular}

a The EXX corresponds to the expansion using the case III parameters of Table II.

shows that the calculation guarantees the convergence of the total energy for these atoms.

\section{Discussion and Final Remarks}

In previous sections we have discussed how the implementation of the EXX formalism in the atom code leads to good results for describing the electronic properties of several atomic systems.

The choice of atom code lies in the great feasibility of this program to be used in the calculation of atoms, because its working philosophy is fairly

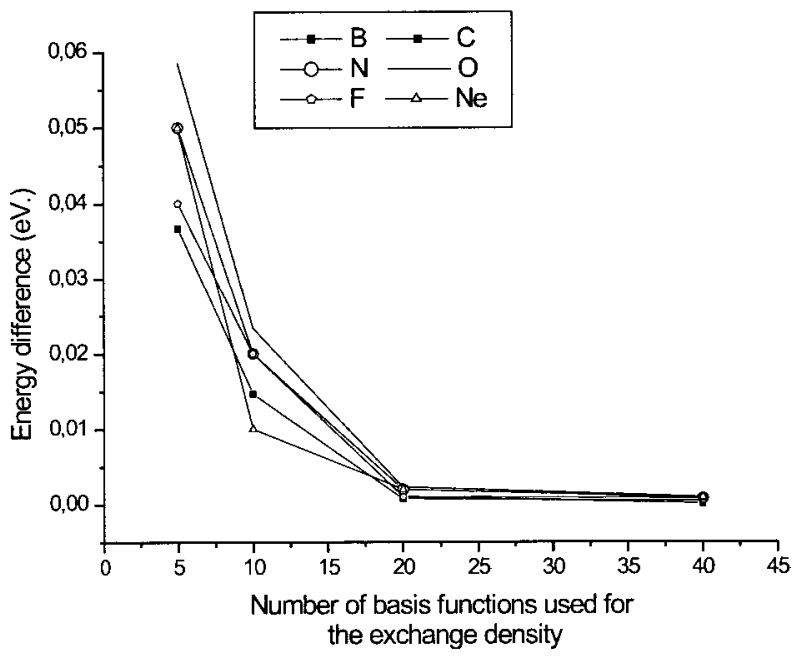

FIGURE 3. Representation of the difference between the total energy calculated and experimental values for the $\mathrm{C}, \mathrm{Ga}, \mathrm{As}$, and $\mathrm{Ti}$ atoms with five different basis set sizes and experimental values as a function of the basis size. The last three points for each atom correspond to the basis set described in Table II. general and because of its ulterior use to molecules and solids. As can be seen in the previous section, the introduction of the EXX exchange term within the Kohn-Sham formalism used allows us to recover a good description of several electronic properties for the systems (such as orbital energies, total energies, and Rydberg series of the atoms).

In the calculation with atom code no basis set is needed for representing the atomic orbitals, because they are obtained by the numerical integration of the Kohn-Sham equations. A Slater type of basis set functions is used to represent the exchange density. This shows that the basis set used to represent the exchange density is independent of the way used to solve the Khon-Sham equations.

We have also confirmed that the EXX method based on a localized basis set expansion of the exchange density leads to a good representation of the atomic properties using a relatively small size of the basis set.

As previously stated, this calculation, also provides an important insight into the influence of the size, and the form of the basis set in more complicated systems, such us solids or molecules.

Our next goal is to implement a more generalized version of this numerical version of the EXX approach in complex systems such as molecules and solids, using the SIESTA code [17].

\section{ACKNOWLEDGVENTS}

The authors thank Prof. A. Görling for fruitful discussions. We also are indebted to E. Artacho, P. Ordejón, D. Sánchez-Portal, J. M. Soler, and A. García for providing a copy of SIESTA that has let us to implement the EXX code in this atomic part: 
the atom code. This work was partially supported by Plan Nacional de I+D of Spain and FEDER programs under references TIC2000-1339-C02-02 and 2FD97-0332-C03-01 and the European Commission contract ENK6 CT200 00310.

\section{References}

1. Parr, R. G.; Yang, W. Density Functional Theory of Atoms and Molecules; Oxford University Press: Oxford, 1989.

2. Hohember, P.; Khon, W. Phys Rev 1964, 136, B864.

3. Density Functional Theory, Vol. 337 of NATO ASI Series B; Gross, E. K. U.; Dreizler, R. M., Eds.; Plenum Press: New York, 1995.

4. Dreizler, R. M.; Gross, E. U. K. Density Functional Theory; Springer: Berlin, 1990.

5. Khon, W.; Sham, L. J. Phys Rev 1965, 140, A1133.

6. Perdew, J. P.; Zunger, A. Phys Rev B 1981, 23, 5075.

7. Perdew, J. P.; Burke, K.; Ernzerhoff, M. Phys Rev Letters 1996, 77, 3865.

8. Perdew, J. P.; Burke, K.; Wang, Y. Phys Rev B 1996, 54, 16533.
9. Talman, J. D.; Shadwick, W. F. Phys Rev A 1976, 14, 36. 10. Krieger, J. B.; Li, Y.; Iafrate, G. J. Phys Rev A 1992, 45, 101.

11. Li, Y.; Krieger, J. B.; Iafrate, G. J. Phys Rev A 1993, 47, 165.

12. Li, Y.; Krieger, J. B.; Iafrate, G. J. Chem Phys Letters 1992, 191, 38

13. Engel, E.; Bosco, S. H. Phys Rev A 1993, 47, 2800.

14. Görling, A. Phys Rev Letters 1996, 77, 3865.

15. Görling, A. Phys Rev B 1996, 53, 7024.

16. Seidili, A.; Görling, A.; Vogl, P.; Majewski, J. A.; Levy, M. Phys Rev B 1994, 50, 196; Int Journal Quamt Symp 1995, 29, 95; Görling, A.; Ley, M. Phys Rev A 1995, 52, 4493; Görling, A. ibid 1996, 54, 3912.

17. Ordejón, P.; Artacho, E.; Soler, J. M. Phys Rev B 1996, 53, 10441; Sánchez-Portal, D.; Ordejón, P.; Artacho, E.; Soler, J. M. Int Journal of Quantum Chem 1997, 65, 453.

18. Quantum Theory of Angular Momentum, Varshalovich, D. A.; Moskalev, A. N.; Khersonskii, V. K., First reprint; Word Scientific, 1999.

19. Grabo, T.; Gross, E. U. K. Chem Phys Letters 1995, 240, 141.

20. Davidson, E. R.; Hagstron, S. A.; Chakavorsky, S. J.; Umar, V. M.; Froese Fisher, C. Phys Rev A 1991, 44, 7071.

21. See Handbook of Chemistry and Physics. David R. Lide $80^{\text {th }}$ edition; CRC Press, 1999-2000. 